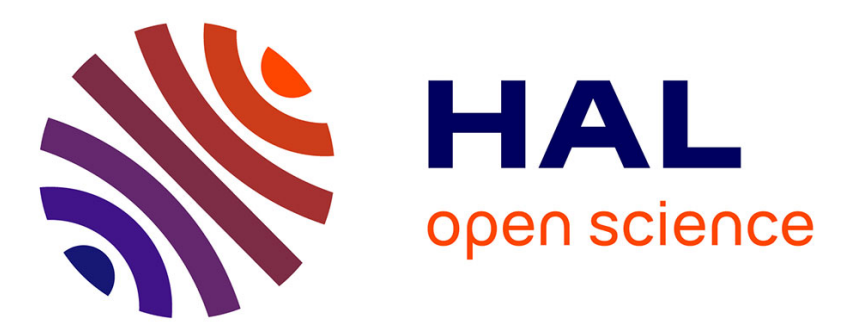

\title{
Multifractality and nonlinear diamagnetic susceptibility in a superconducting network at percolation
}

\author{
Alex Hansen, Stéphane G. Roux
}

\section{To cite this version:}

Alex Hansen, Stéphane G. Roux. Multifractality and nonlinear diamagnetic susceptibility in a superconducting network at percolation. Journal de Physique, 1988, 49 (8), pp.1379-1385. 10.1051/jphys:019880049080137900 . jpa-00210818

\section{HAL Id: jpa-00210818 https://hal.science/jpa-00210818}

Submitted on 1 Jan 1988

HAL is a multi-disciplinary open access archive for the deposit and dissemination of scientific research documents, whether they are published or not. The documents may come from teaching and research institutions in France or abroad, or from public or private research centers.
L'archive ouverte pluridisciplinaire HAL, est destinée au dépôt et à la diffusion de documents scientifiques de niveau recherche, publiés ou non, émanant des établissements d'enseignement et de recherche français ou étrangers, des laboratoires publics ou privés. 


\title{
Multifractality and nonlinear diamagnetic susceptibility in a superconducting network at percolation
}

\author{
Alex Hansen (*) and Stéphane Roux ( $\left.{ }^{1}\right)$ \\ Groupe de Physique des Solides de l'Ecole Normale Supérieure, 24 rue Lhomond, 75231 Paris Cedex 05, \\ France \\ (1) Laboratoire de Physique de la Matière Hétérogène, Ecole Supérieure de Physique et Chimie Industrielles, \\ 10 rue Vauquelin, 75231 Paris Cedex 05, France
}

(Reçu le 10 mars 1988, accepté le 19 avril 1988)

\begin{abstract}
Résumé. - Nous avons étudié, par simulations numériques, la distribution des courants dans un réseau bidimensionnel de jonctions Josephson dans un champ magnétique. Au seuil de percolation, dans l'approximation de jonctions linéaires, cette distribution est multifractale. Nous avons aussi considéré la première correction non linéaire de la susceptibilité diamagnétique. Cette correction non linéaire suit une loi d'échelle dont l'exposant gouverne un moment de la distribution des courants dans le modèle linéaire. Le champ magnétique pour lequel cette correction devient importante, varie selon une loi de puissance de la susceptibilité dont l'exposant est donné par une combinaison d'exposants du spectre multifractal du modèle linéaire.
\end{abstract}

\begin{abstract}
We have calculated numerically the current distribution of a two-dimensional Josephson junction network in a magnetic field. At the percolation threshold and in the approximation of linear junctions this distribution is multifractal. We have also considered the lowest order nonlinear corrections to the diamagnetic susceptibility. This correction scales with one of the exponents of the multifractal exponents appearing in the linear approximation. The crossover magnetic field at which the nonlinear corrections become important scales with a power law in the susceptibility. The exponent of this power law is given by a combination of multifractal exponents of the linearized approximation.
\end{abstract}

\section{Introduction.}

The critical properties of the diamagnetic susceptibility of disordered lattices of superconductors near the percolation threshold have been extensively studied a few years ago [1-4]. These, and later studies [5], have, however, always treated this problem in a linearized approximation, thus eliminating the effects of any weak junctions between the various superconducting elements constituting the superconducting lattice. In this paper we study the lowest order nonlinear corrections to the diamagnetic susceptibility, assuming that the junctions are dominated by the Josephson effect. We show that scaling exponents describing the behaviour of these nonlinear corrections are combinations of the multifractal scaling exponents of the moments of the

(*) Present address : Institut für Theoretische Physik, Universität zu Köln, 5000 Köln 41, F.R.G. current distribution in the network in the linear approximation.

These questions have gained new relevance with the discovery of superconductivity at high temperatures in certain ceramic materials. It was first believed that the Josephson junctions which appeared to be present in these materials stemmed from weak contacts between superconducting grains. However, now mounting evidence points towards these Josephson junctions appearing inside the superconducting grains. There the supercurrent flows two-dimensionally, and it has been suggested [6] that one is dealing with a two-dimensional network of Josephson junctions near the percolation threshold. Since we here determine exponents associated with the linear and nonlinear behaviour of the susceptibility, this work may be relevant for the experimental testing of the above picture of high$T_{\mathrm{c}}$ superconductivity.

Close enough to the percolation threshold, $p_{\mathrm{c}}$, the 
diamagnetic susceptibility $\chi$ diverges with a power law of the form [7]

$$
\chi \sim\left|p-p_{\mathrm{c}}\right|^{-b} .
$$

This power law is valid in the linearized approximation of the equations governing the currents flowing in a disordered lattice of superconductors. There have been two suggestions relating the exponent $b$ to the exponents appearing in the random resistor network, namely [7]

$$
b=2 \nu-t,
$$

where $\nu$ is the critical exponent of the correlation length, $\xi$, and $t$ is the conductivity critical exponent, or [2]

$$
b=2 \nu-t+\beta,
$$

where $\beta$ is the critical exponent governing the vanishing of the density of the infinite cluster. Our previous study [5], as well as the results of the present paper, strongly support the first suggestion.

In the linear approximation, the diamagnetic susceptibility is related to the second moment of the distribution of currents flowing in the superconducting network due to the presence of a (small) uniform magnetic field at $p_{\mathrm{c}}$. Since this moment is scaling with a non-trivial exponent $b$, it is natural to ask whether the other moments of this current distribution also show non-trivial scaling, thus revealing a multifractal structure [8]. It turns out that this is indeed so. Thus, we find that the $n$-th current moment scales as

$$
\sum i_{k}^{n} \sim L^{2+b(n) / \nu}
$$

where $i_{k}$ is the current through the $k$-th bond in the network and $2+b(n) / \nu$ is the scaling exponent with respect to the linear size of the lattice, $L$. The fractal dimension of the current-carrying subset of bonds, $2+b(0) / \nu$, is 2 , a result which is of no surprise ; all closed loops and not just those on the percolating cluster carry a current. Furthermore, $b(2)=b$, the exponent appearing in equation (1).

Close to the critical point, $p_{c}$, and when the magnetic field is small, the linear terms in the governing equations dominate. However, at some crossover magnetic field $B_{\mathrm{c}}$ to be defined later, the nonlinear corrections become important. As was earlier shown in the nonlinear random resistor network [9], we also here find that $B_{\mathrm{c}}$ scales with the diamagnetic susceptibility to some non-trivial power. More specifically, if $L$ is the linear size of the network, we find that

and

$$
B_{\mathrm{c}}(L) \sim \chi_{0}(L)^{-y}, \quad L \ll \xi
$$

$$
B_{\mathrm{c}}(L) \sim\left(L^{2} \chi_{0}(L)\right)^{x}, \quad L \gg \xi
$$

where $y$ and $x$ are exponents related to the exponents of the moments of the current distribution at nonzero magnetic field, i.e. $b(n) / \nu$. The subscript 0 in the susceptibility indicates that this is the one found in the linear approximation.

In section 2 we describe our model. In section 3 we demonstrate the multifractal structure of the current distribution. In section 4, we discuss the nonlinear corrections to the diamagnetic susceptibility. We conclude in section 5 with a brief summary of our results.

\section{The model.}

We consider a two-dimensional square lattice of size $L \times(L+1)$ where the bonds are either superconducting with probability $p$ or normal with probability $1-p$. The bonds are thought to be long and thin compared to the coherence length defined by the Ginzburg-Landau equations, so that it does not matter whether the normal bonds are metallic or insulators. Furthermore, we assume that the superconducting bonds consist of two superconducting parts joined by a Josephson junction. At each node we define a phase angle $\phi_{i}$. B is a uniform magnetic field imposed perpendicular to the plane of the lattice, and $A$ is a vector field which generates $B$. We have chosen a gauge such that $A$ has a non-zero component (which is linearly increasing) only in the direction one axis of the $L \times(L+1)$ lattice. This is shown in figure 1 . The current running between node $i$ and its neighbour $j$ (assuming that they are connected by a superconducting bond) is [10]

$$
j_{i j}=e_{i j} \sin \left(\phi_{i}-\phi_{j}-A_{i j}\right)
$$

where $A_{i j}$ is the circulation of $A$ along the bond $i-j$, i.e. $A_{i j}=\int_{i}^{j} A \mathrm{~d} l$. To simplify the notation for
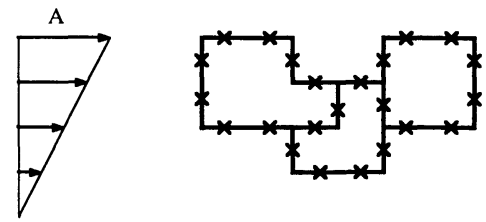

$\odot$ B

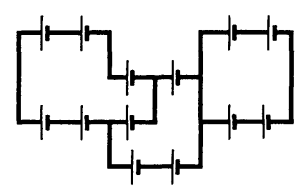

Fig. 1. - An example of the network of superconductors (bonds) and Josephson junctions (crosses), is indicated in the upper right part of the figure. The imposed magnetic field $B$ and the corresponding vector potential $A$ is shown in the upper left part. The equivalent random resistor network with current sources in series with the resistors (indicated as bonds), is indicated in the lower left part of the figure. 
later we introduce $e_{i j}$ which is one if $i$ and $j$ are linked by a superconducting bond, and zero otherwise. The Hamiltonian of the lattice reads

$$
H=-\sum_{(i, j)} e_{i j} \cos \left(\phi_{i}-\phi_{j}-A_{i j}\right)
$$

where the sum runs over all pairs $(i, j)$. For a given magnetic field, $B$, the energy $E$ is the minimum of $H$ with respect to the phases $\phi_{i}$, and the susceptibility is

$$
\begin{aligned}
\chi= & (1 / S) \partial^{2} E / \partial B^{2} \\
= & (1 / S) \sum_{(i, j)} e_{i j}\left(\cos \psi_{i j}\left(\partial \psi_{i j} / \partial B\right)^{2}+\right. \\
& \left.+\sin \psi_{i j}\left(\partial^{2} \psi_{i j} / \partial B^{2}\right)\right)
\end{aligned}
$$

where $S$ is the area of the (full) lattice, and

$$
\psi_{i j} \equiv \phi_{i}-\phi_{j}-A_{i j}
$$

The phases $\phi_{i}$ are here the ones that minimizes the Hamiltonian, and it is found by solving the nonlinear Kirchhoff equations, [1]

$$
\sum_{i(n n)} e_{i i(n n)} \sin \left(\phi_{i}-\phi_{i(n n)}-A_{i i(n n)}\right)=0
$$

where $i(n n)$ is a nearest neighbour node of node $i$.

We notice that for $B=0$, there may still be currents flowing, as a result of the periodicity in the current-phase relation [11], a property that gives rise to a difficult spin-glass problem. We assume here, however, that the variations in the phases from node to node are small enough so that this situation does not appear. We also assume that the temperature of the network is so low that current-vortices may not be generated by thermal fluctuations.

Let us now expand equations (7) and (9) to the third order in $B$, by assuming the following expansion of $\psi_{i j}$;

$$
\psi_{i j}=\psi_{i j}^{(1)} B+\psi_{i j}^{(3)} B^{3}+\cdots
$$

as the even terms are zero since $\psi_{i j}$ is an odd function of $B$. To this order the Kirchhoff equations become

$$
\begin{aligned}
\sum_{i(n n)} e_{i i(n n)}\left(\psi_{i i(n n)}^{(1)} B+\psi_{i i(n n)}^{(3)} B^{3}-\right. \\
\left.-(1 / 6)\left(\psi_{i i(n n)}^{(1)}\right)^{3} B^{3}+\cdots\right)=0 .
\end{aligned}
$$

Furthermore, the diamagnetic susceptibility becomes

$$
\begin{aligned}
\chi= & (1 / S) \sum_{(i, j)} e_{i j}\left(\left(\psi_{i j}^{(1)}\right)^{2}+12 B^{2} \psi_{i j}^{(1)} \psi_{i j}^{(3)}-\right. \\
& \left.-(1 / 2) B^{2}\left(\psi_{i j}^{(1)}\right)^{4}+\cdots\right) .
\end{aligned}
$$

Now, solving the Kirchhoff equations, equation (11), to the lowest order in $B$, amounts to solving the Kirchhoff equations for a standard random resistor network, with the only difference that the terms $A_{i j}$ act as external voltage sources in series with each resistor in the network, as illustrated in figure 1 :

$$
\begin{aligned}
\sum_{i(n n)} e_{i i(n n)} \psi_{i i(n n)}^{(1)} & =\sum_{i(n n)} e_{i i(n n)} \times \\
\times & \left(\phi_{i}^{(1)}-\phi_{i(n n)}^{(1)}-A_{i i(n n)}\right)=0 .
\end{aligned}
$$

Thus, we find the $\psi_{i j}^{(1)}$. These phases can now be plugged back into the third-order expansion of the Kirchhoff equations, equation (11), and we end up with an equation of the form

$$
\sum_{i(n n)} e_{i i(n n)}\left(\psi_{i i(n n)}^{(3)}-(1 / 6)\left(\psi_{i i(n n)}^{(1)}\right)^{3}\right)=0,
$$

and we notice that this equation has the same form as the one that was needed to find the linear solution, equation (13) :

$$
\sum_{i(n n)} e_{i i(n n)}\left(\phi_{i}^{(3)}-\phi_{i(n n)}^{(3)}-(1 / 6)\left(\psi_{i i(n n)}^{(1)}\right)^{3}\right)=0
$$

if one replaces the source term $A_{i i(n n)}$ in equation (13) by $(1 / 6) \psi_{i i(n n)}^{(3)}$. Thus, in the random resistor network analogy, the problem is the same, only the voltage sources in series with each of the resistors have changed. We note that this perturbative method is not limited to third order only, but may be repeated to any order. We limit ourselves here to only studying the leading nonlinear correction.

We solved these equations numerically by using the Conjugate Gradient method. The presence of the voltage sources in the Kirchhoff equations made Fourier acceleration difficult [12]. The data we present in the following are based on 2500 samples of size $L=16,1000$ samples of size $L=20$, 1000 samples of size $L=25,1000$ samples of size $L=32,2500$ samples of size $L=40,202$ samples of size $L=50,1000$ samples of size $L=64$, and 336 samples of size $L=100$.

\section{Multifractal structure in the linear regime.}

The random resistor network provides an excellent example of multifractality [8]. Suppose the random resistor network is hooked up to an external current source, so that the ports are a distance $L$ apart, for example by short-circuiting separately the upper and lower edges of the lattice by using bus bars. The resulting currents, which only flow through the backbone of the spanning cluster (i.e. of the incipient infinite cluster in percolation language), will have a multifractal distribution near the percolation threshold, $p_{\mathrm{c}}$, if the correlation length $\xi \sim$ $\left|p-p_{\mathrm{c}}\right|^{-\nu}$ is larger than $L$. One finds for the $n$-th moment of the current distribution (when $n \leqslant 0$, see Ref. [13]) :

$$
\sum_{(i, j)} e_{i j}\left(i_{i j}\right)^{n} \sim L^{x(n)}
$$


where $i_{i j}$ is the current through the bond $i-j$. Each of the exponents $x(n)$ is independent from the others in the sense that no algebraic expression is known between them. This infinite hierarchy of exponents provides a complete description of the structure of the current-carrying backbone near the percolation threshold. Besides this, some of these exponents are directly related to measurable quantities in the random resistor network : $x(0)$ is the fractal dimension of the current-carrying backbone, $x(2)$ is $t / \nu$, i.e. describes the scaling of the conductance of the backbone, and $x(4)$ is related to the scaling of the $(1 / f)$ noise that is always present in resistor networks.

These exponents $x(n)$ are calculated by generating ensembles of backbones of different sizes, keeping some physical quantity such as the voltage drop across the backbone, or the total current flowing in it constant. Each type of ensemble will give a different set of exponents, but they are related through simple algebraic combinations [13]. In this paper $x(n)$ refers to exponents found in the constant-current ensemble. In the same way, one may find different exponents $b(n) / \nu$ in the superconducting network depending on which ensemble one averages over. Here we have used the constant magnetic-field ensemble, but other ensembles are possible.

The set of currents resulting from a weak magnetic field (so that the linearized Kirchhoff equations are a good approximation) in the superconducting network also form a multifractal distribution. As in the random resistor network, we mean by this that for the moments of the current distribution,

$$
\sum_{(i, j)} e_{i j}\left(\psi_{i j}^{(1)}\right)^{n} \sim L^{2+b(n) / \nu} .
$$

There are two main differences between the currents in the random resistor network and the currents in the superconducting network: (1) the currents are not limited to only some backbone of the incipient infinite cluster, they exist in all loops in all clusters of connected bonds; (2) there are external voltage sources everywhere in the network, and not just two, a distance $L$ apart. These two differences account for the difference between $b(n) / \nu$ and $x(n)$.

In table I we give the scaling exponents for the even moments up to the tenth one of the current distribution. They were calculated by least-square fits to the moments from $L=16$ to $L=100$. In figure 2 we plot the zeroth, second and fourth moments, and in figure 3 the sixth, eight and tenth moments, all versus $L$. In figure 4 we plot the

Table I. - The scaling exponents $2+b(n) / \nu$ and $2+c(n) / \nu$ for the superconducting network. We have also listed the corresponding (constant-current ensemble) random resistor network exponents for comparison.

\begin{tabular}{|c|c|c|c|}
\hline$n$ & $2+b(n) / \nu$ & $2+c(n) / \nu$ & $x(n)$ \\
\hline 0 & 2 & 2 & $1.62(2)$ \\
\hline 2 & $3.03(2)$ & $1.07(2)$ & $0.97(1)$ \\
\hline 4 & $4.75(5)$ & $0.84(5)$ & $0.81(2)$ \\
\hline 6 & $6.68(6)$ & $0.81(6)$ & $0.77(3)$ \\
\hline 8 & $8.61(6)$ & $0.79(6)$ & $0.74(2)$ \\
\hline 10 & $10.54(4)$ & $0.76(4)$ & 0.75 \\
\hline
\end{tabular}

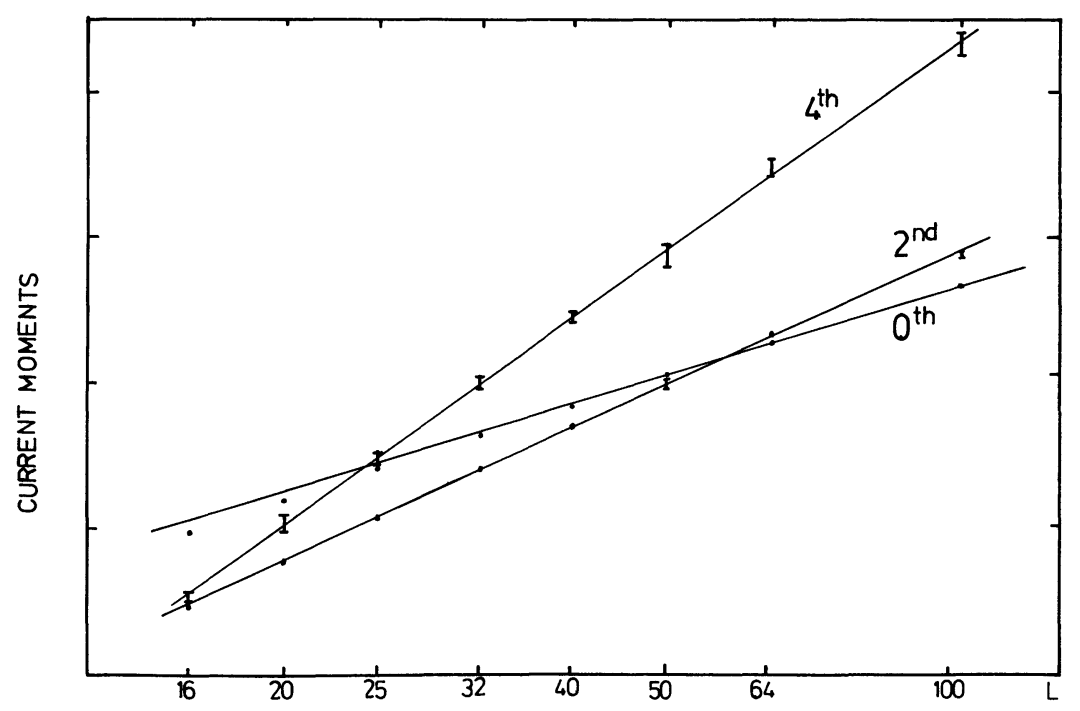

Fig. 2. - The zeroth, second and fourth moment plotted against the linear size of the lattices, $L$. 


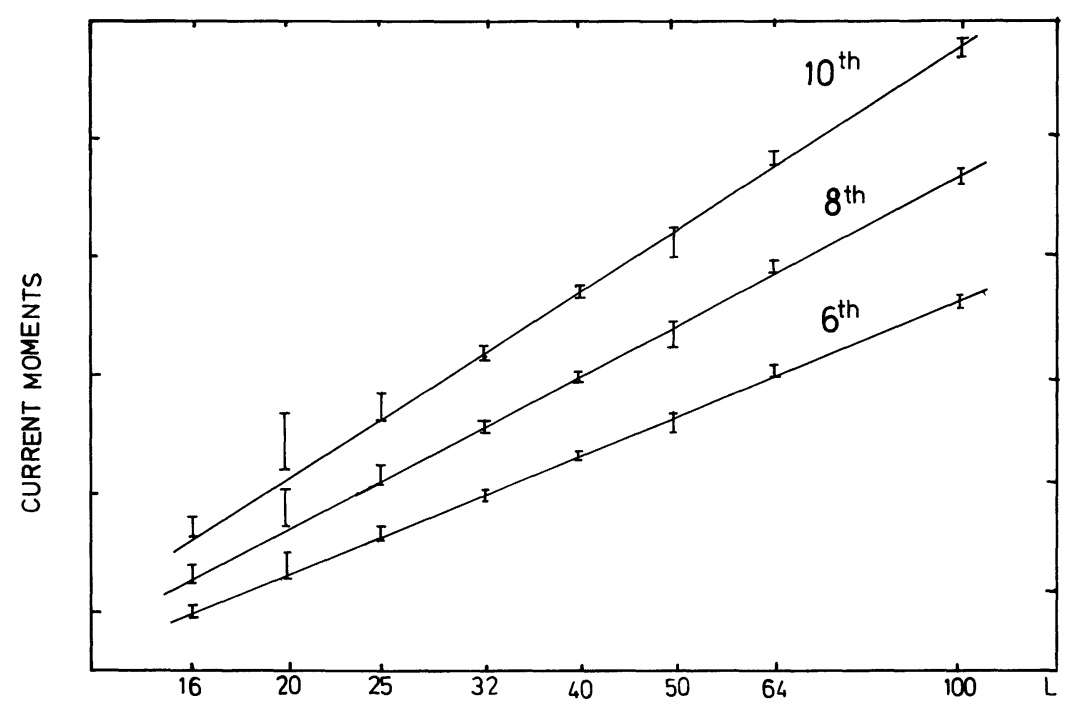

Fig. 3. - The sixth, eight and tenth moment plotted against the linear size of the lattice $L$.

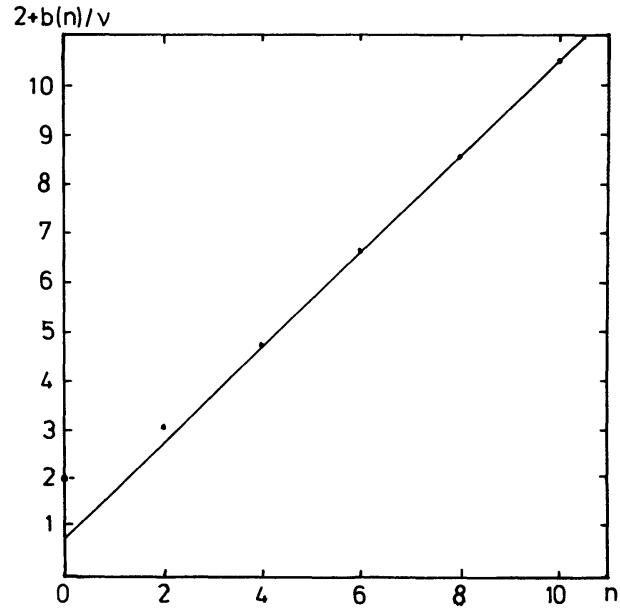

Fig. 4. - The exponents $2+b(n) / \nu$ as a function of $n$. The straight line is given by $1 / \nu+n t / \nu$.

exponents $2+b(n) / \nu$ as a function of $n$. It seems that for large $n$ (but not for small $n$ ), the exponents behave as

$$
2+b(n) / \nu=1 / \nu+n t / \nu .
$$

This indicates that an ensemble different from the constant-magnetic field one may be constructed such that the exponents tend towards a constant for large $n$ : the $n$-th moment for each realization should be divided by the second moment to the $n /(1-4 \nu / t)$ power. This leads to a new series of exponents for the superconducting network given by the equation

$$
c(n) / \nu=b(n) / \nu-n t / \nu
$$

where $c(0) / \nu=0, c(2) / \nu=2-3 t / \nu$ and $c(n) / \nu$ asymptotically approaches $1 / \nu-2[14]$. The values of these exponents are also given in table $I$.

We note that there does not seem to be any simple relation connecting the random resistor exponents $x(n)$ and the exponents $c(n)$ for general $n$.

\section{Scaling of the onset of non-linearity.}

We now turn to the question of the width of the linear regime where one may measure $\chi_{0} \sim$ $\left|p-p_{\mathrm{c}}\right|^{-b}$ in terms of the exterior magnetic field $B$. Following Gefen et al. [9], we define a crossover magnetic field, $B_{c}$, as the field at which

$$
\chi \sim \chi_{0}(1+\varepsilon)
$$

where $\varepsilon$ is a small fixed number. As in section 1 , $\chi_{0}$ is the linearized susceptibility, and $\chi$ is the total susceptibility.

Suppose now that we are close enough to the percolation threshold so that $\xi \gg L$. The susceptibility with the lowest order nonlinear corrections, equation (12), can then be written as

$$
\begin{aligned}
\chi=(1 / S)\left(a L^{b / \nu+2}\right. & +12 B^{2} \sum_{(i, j)} e_{i j} \psi_{i j}^{(1)} \psi_{i j}^{(3)}- \\
& \left.-(1 / 2) B^{2} c L^{b(4) / \nu+2}\right)
\end{aligned}
$$

where $\chi_{0} \sim(a / S) L^{b / \nu+2}$. The sum $\sum_{(i, j)} e_{i j} \psi_{i j}^{(1)} \psi_{i j}^{(3)}$ is zero as can be seen as follows :

$$
\begin{aligned}
\sum_{(i, j)} e_{i j} \psi_{i j}^{(1)} \psi_{i j}^{(3)} & =\sum_{(i, j)} e_{i j} \psi_{i j}^{(1)}\left(\phi_{i}^{(3)}-\phi_{j}^{(3)}\right) \\
& =2 \sum_{(i, j)} e_{i j} \psi_{i j}^{(1)} \phi_{i}^{(3)}=0,
\end{aligned}
$$

where we have used that $\psi_{i j}^{(1)}=-\psi_{j i}^{(1)}$, and Kirchhoff's law, equation (13). Thus, we have

$$
\begin{aligned}
\chi & =(1 / S)\left(a L^{b / \nu+2}-(1 / 2) B^{2} c L^{b(4) / \nu+2}\right) \\
& =\chi_{0}\left(1+B^{2} c^{\prime} L^{(b(4)-b) / \nu}\right) .
\end{aligned}
$$

Comparing this equation with equation (20) then determines the crossover magnetic field in terms of 
$L$, and thus also in terms of $\chi_{0}$ :

$$
\varepsilon=B_{\mathrm{c}}^{2} c^{\prime} L^{(b(4)-b) / \nu}=B_{\mathrm{c}}^{2} c^{\prime \prime} L^{(b(4)-b) / b}
$$

and thus, since $\varepsilon$ is a fixed number

$$
B_{\mathrm{c}}(L) \sim \chi_{0}^{-(b(4)-b) / b}=\chi_{0}^{-y}
$$

as quoted in equation (3).

A much more realistic case from an experimental point of view is when $L \gg \xi$. In a uniform system the diamagnetic susceptibility scales as $L^{2}$. The network we are dealing with here is uniform on length scales larger than the correlation length. Thus we expect the diamagnetic susceptibility to scale as

$$
\chi(L) \sim(L / \xi)^{2} \chi(\xi) .
$$

Thus, we have that

$$
\begin{aligned}
B_{\mathrm{c}}(L) \sim \chi_{0}(\xi)^{-y} & \sim \xi^{-y b / \nu} \sim \\
& \sim\left(\chi_{0}(\xi) / \xi^{2}\right)^{-y b /(b-2 \nu)} .
\end{aligned}
$$

Now, using equation (25), this becomes

$$
\begin{aligned}
& B_{\mathrm{c}}(L) \sim\left(\chi_{0}(L) / L^{2}\right)^{-y b /(b-2 \nu)} \sim \\
& \sim\left(\chi_{0}(L) / L^{2}\right)^{x} .
\end{aligned}
$$

Thus, we find $x=(b-b(4)) / 2(b-2 \nu)$ for the scaling exponent for the crossover field in terms of the measured diamagnetic susceptibility of a network close to the percolation threshold, but where the correlation length is smaller than the network size. Using the values for the exponents found in section 3, we find that $x \approx 0.89$ and $y \approx 1.67$.

It should be noticed that the new exponents introduced in this section for describing the lowestorder nonlinear corrections to the susceptibility are combinations of the multifractal scaling exponents, $b(n)$, occurring in the linear approximation. This may be a general result valid to all order in the expansion in $B$ of equation (9). If this is so, the entire hierarchy of exponents, $b(n)$, would have direct physical meaning.

\section{Conclusion.}

The current distribution near the percolation threshold in a disordered network of superconducting Josephson junctions, and which results from a weak external and uniform magnetic field, shows multifractal behaviour. We have calculated some of the exponents belonging to the infinite hierarchy of exponents. They seem to approach a simple asymptotic behaviour.

The presence of the Josephson junctions makes the problem nonlinear. We have studied the effect of this nonlinearity on the diamagnetic susceptibility to the lowest order in the external magnetic field. This correction scales with the scaling exponent of the fourth-order moment of the current distribution in the linear approximation. Of special interest is a suitably defined crossover magnetic field at which the nonlinear corrections become important. This crossover magnetic field scales with the measured linear diamagnetic susceptibility with two different scaling exponents, one when the sample is much larger than the percolation correlation length, and another when the sample is much larger than the correlation length (but the correlation length is large). These scaling exponents are related to the multifractal scaling exponents of the moments of the current distribution in the network in the linear approximation.

Usually, the multifractal spectrum is rather unaccessible from an experimental point of view : only a very few (basically the zeroth, second and fourth) moments of the current distribution have simple interpretations in terms of easily measurable macroscopic quantities. However, in this case there seem to be a connection between the multifractal spectrum and the behaviour of the nonlinear corrections to the diamagnetic susceptibility. We have shown here that this is so for the lowest-order nonlinear corrections. Thus, this system may provide a multifractal spectrum which is directly measurable by experiments.

These results should also be relevant to the ongoing study of the new high- $T_{\mathrm{c}}$ superconductors, as mentioned in the introduction. There is now strong evidence that these are highly heterogeneous materials, perhaps close enough to the percolation threshold to possess a large correlation length [6]. A scaling behaviour of the necessary magnetic field to produce a crossover in the scaling behaviour of the diamagnetic susceptibility would support the existence of such a correlation length.

This study has been done for two-dimensional systems. It ought to be redone for the three-dimensional case. Qualitatively different results may be found ; for example that the corresponding $b / \nu$ exponent has the opposite sign in three dimensions as compared to its value in two dimensions.

\section{Acknowledgments.}

We wish to thank E. Guyon, E. L. Hinrichsen, M. A. Novotny and D. Stauffer for useful discussions on this subject. A. H. benefitted greatly from the hospitality of J. Feder and T. Jøssang at the University of Oslo where a large part of this work was done. It was completed at IBM Bergen Scientific Centre due to the hospitality of M. Novotny. The computations were done on an FPS-164 computer at the Ecole Normale Supérieure funded by GRECO 70 (Experimentation numérique) and on an IBM 3090 at IBM Bergen Scientific Centre. A. H. was supported by CEN-Saclay through a Joliot-Curie fellowship and S. R. by the Ecole Nationale des Ponts et Chaussées. 


\section{References}

[1] Alexander, S., Phys. Rev. B 27 (1983) 1541.

[2] Rammal, R., Lubensky, T. C. and Toulouse, G., Phys. Rev. B 27 (1983) 2820 ; J. Phys. Lett. France 44 (1983) L-65.

[3] Rammal, R. and Angles d'Auriac, J. C., J. Phys. C 16 (1983) 3933.

[4] Bowman, D. R. and Stroud, D., Phys. Rev. Lett. 52 (1984) 299.

[5] Roux, S. and Hansen, A., Europhys. Lett. 5 (1988) 473.

[6] Deutscher, G. and Müller, K. A., Phys. Rev. Lett. 59 (1987) 1745.

[7] De Gennes, P. G., C.R. Hebd. Séan. Acad. Sci. Ser. II 292 (1981) 9.

[8] De Arcangelis, L., Redner, S. and Coniglio, A., Phys. Rev. B 31 (1985) 4725 ;

Rammal, R., Tannous, C., Breton, P. and Tremblay, A.-M. S., Phys. Rev. Lett. 54 (1985) 1718.
[9] Gefen, Y., Shih, W.-H., Laibowitz, R. B. and Viggiano, J. M., Phys. Rev. Lett. 57 (1986) 3097.

[10] De Gennes, P. G., Superconductivity of Metals and Alloys (Benjamin, New York) 1966.

[11] Jacobs, L., 'José, J. V., NovotnY, M. A. and Goldman, A. M., Europhys. Lett. 3 (1987) 1295 and reference therein.

[12] Batrouni, G. G., Hansen, A. and Nelkin, M., Phys. Rev. Lett. 57 (1986) 1336 ;

Batrouni, G. G. and Hansen, A., submitted to $J$. Stat. Phys.

[13] Batrouni, G. G., Hansen, A. and Roux, S., submitted to Phys. Rev. A.

[14] A word of caution about the limiting values of the exponents in the limit of large $n$ has been given in BAtrouni, G. G., Hansen, A. and Nelkin, M., J. Phys. France 48 (1987) 771. 